\title{
Venipuncture-Related Median Nerve Palsy Disguised as Intraoperative Brachial Plexus Injury
}

\author{
Lisa B.E. Shields ${ }^{a}$ Brandon Sutton $^{b}$ Vasudeva G. Iyer ${ }^{c}$ \\ Christopher B. Shields ${ }^{a, d} \quad$ Abigail J. Rao $^{a}$ \\ a Norton Neuroscience Institute, Norton Healthcare, Louisville, KY, USA; ${ }^{\text {b} O h i o ~ V a l l e y ~ P a i n ~}$ \\ Institute, Louisville, KY, USA; ' Neurodiagnostic Center of Louisville, Louisville, KY, USA; \\ ${ }^{\mathrm{d} D e p a r t m e n t}$ of Neurological Surgery, University of Louisville School of Medicine, Louisville, \\ KY, USA
}

\section{Keywords}

Venipuncture - latrogenic median nerve - Brachial plexus - Somatosensory evoked potential . Ultrasound

\begin{abstract}
latrogenic peripheral nerve injuries may result from transection, stretch, compression, injections, ligature, heat, anticoagulant use, and radiation. latrogenic median nerve palsy has been reported rarely. We report a case of a woman who underwent craniectomy for treatment of trigeminal neuralgia. Intraoperatively, a transient decline in the amplitude of the left upper extremity somatosensory evoked potentials (SSEPs) was noted. This finding was presumed to be due to the traction on the brachial plexus as it improved with repositioning. Immediately upon waking from anesthesia, the patient experienced sensorimotor deficits in the left median nerve distribution. Ecchymoses from venipuncture were observed in this area. Electrodiagnostic studies confirmed a left median nerve neuropathy localized in the antebrachial area. Neurosurgeons and neurologists should be alert to potential iatrogenic median nerve palsy following vascular access at the antebrachial region. Vascular access could be performed under the ultrasound guidance when a patient is under anesthesia or unable to give sensory feedback. Furthermore, placing an additional recording electrode over the proximal upper arm during intraoperative SSEP monitoring aids in distinguishing between brachial plexus and peripheral nerve injuries.
\end{abstract}


Shields et al.: Venipuncture-Related Median Nerve Palsy

\section{Introduction}

Iatrogenic nerve injuries may arise from direct damage during surgical trauma, pressure, or traction from malpositioning during anesthesia, injection of neurotoxic substances, and radiation [1, 2]. In an extensive retrospective study spanning a period of 10 years, the frequency of perioperative peripheral nerve injuries was $0.03 \%$ [3]. These injuries represent $17.4 \%$ of all peripheral nerve traumas [2], and the leading cause is inappropriate patient positioning under general anesthesia [4].

Whereas venipuncture is a common invasive medical procedure that is usually devoid of complications, rarely, it may cause peripheral nerve injury. The incidence of nerve injuries associated with neuropathic pain following venipuncture ranges between 1 in 21,000 and 31,000 and may involve routine venipuncture for blood donation or intravenous infusion [5-8]. Difficulty accessing the vein and multiple attempts increase the risk of developing a peripheral nerve injury $[7,8]$. Venipuncture-related nerve injuries have been described, including the medial and lateral antebrachial cutaneous nerves, radial nerve, and median nerve $[5,6,8]$. The prognosis of venipuncture-induced neuropathic pain is good when there is no development of complex regional pain syndrome [5, 9]. It has been reported that 70 and $96 \%$ of venipuncture-related nerve injuries resolve within 1 to 6 months, respectively [7].

Here, we report a case of iatrogenic median nerve palsy following craniectomy in the three-quarter prone position. Factors distinguishing iatrogenic brachial plexus injury from median nerve injury are discussed, with specific attention to electrodiagnostic and ultrasound (US) findings.

\section{Case Description}

\section{History and Radiological Imaging}

A 29-year-old woman with a history of obesity (BMI $39 \mathrm{~kg} / \mathrm{m}^{2}$ ) and Raynaud's disease presented with medically refractory trigeminal neuralgia. She was indicated for exploration of the trigeminal nerve, with planned internal neurolysis in the absence of neurovascular compression.

\section{Operative Course}

The patient underwent left retrosigmoid craniectomy for exploration and possible internal neurolysis of the trigeminal nerve. Peripheral intravenous (IV) lines were attempted in the hands bilaterally and in the posterior left wrist, none of which were used due to phlebitis. A right radial arterial line was inserted after 2 attempts. After multiple attempts, venous access was obtained in the left antecubital fossa. Only the peripheral IV line in the right hand was placed prior to induction of general anesthesia. A $1 \%$ lidocaine intradermal injection with a $1 \mathrm{~mL}$ dose was used to anesthetize the site prior to attempting venous access.

The patient was positioned in the three-quarter prone position, with her head in Mayfield 3-point fixation to elevate the ipsilateral mastoid process. The dependent arm was stabilized and padded in an arm sling. The superior (left) arm was padded in gentle flexion, with the ipsilateral shoulder taped to the foot of the bed to open the angle between the retromastoid region and shoulder. Neuromonitoring electrodes for somatosensory evoked potentials (SSEPs) of the bilateral upper and lower extremities as well as brainstem auditory evoked potentials and electromyography (EMG) of the facial nerve were inserted. The baseline waveforms were symmetric.

\section{Karger'}




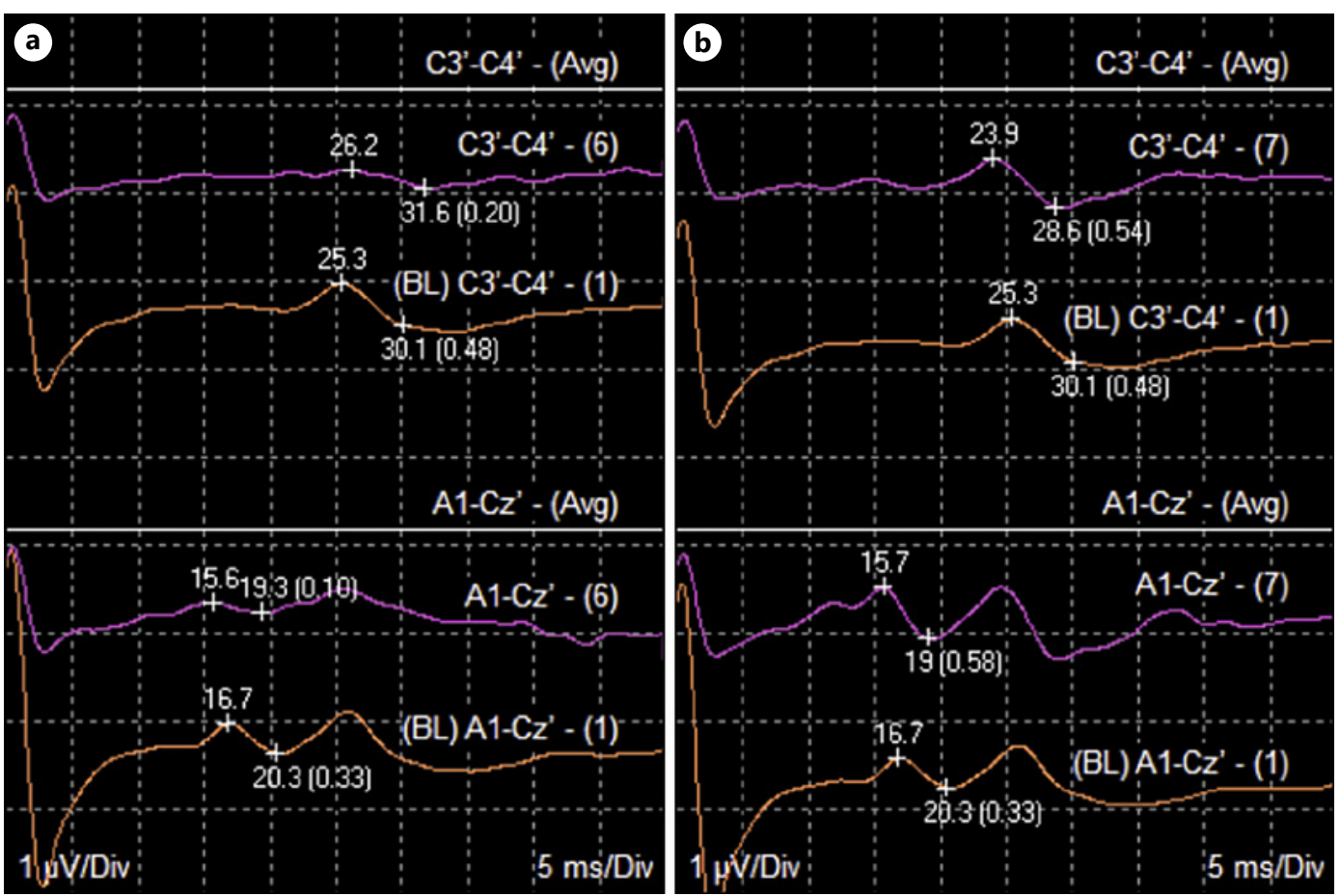

Fig. 1. Intraoperative SSEP tracings showing a decline in the amplitude of the left upper extremity SSEPs (a, purple tracing) as compared with baseline waveforms (a, orange tracing). Upon de-rotating the operative table, cortical and subcortical waveforms (b, purple tracing) returned to baseline (b, orange tracing). In both the panels, top waveforms are cortical traces, and the bottom waveforms are subcortical traces. SSEPs, somatosensory evoked potentials.

Halfway through the posterior fossa exploration, and while the surgical table was tilted rotating the ipsilateral shoulder toward gravity, there was a decline in amplitude of the left upper extremity SSEPs (Fig. 1a, b). The table was subsequently de-rotated to allow the patient's left shoulder to return to a neutral position. TheSSEPs spontaneously and quickly returned to baseline.

\section{Postoperative Findings}

Immediately upon awakening from anesthesia, the patient reported numbness, burning pain, and cold dysesthesia of the left medial forearm with extension to the radial 3 fingers and radial half of the ring finger. On examination, pinprick sensation was diminished over the radial left 3 fingers and radial side of the palm, with loss of 2-point discrimination over the radial 3 fingers of the left hand. There was also flexor pollicis longus (FPL) and flexor digitorum profundus (FDP) weakness of the left index finger. Only the muscles innervated by anterior interosseous nerve (AIN) showed weakness. There was no weakness of the pronator teres, flexor carpi radialis, flexor digitorum superficialis, abductor pollicis brevis (APB), or opponens pollicis. Ecchymosis was noted in the volar aspect of the proximal left forearm 1 week postoperatively (Fig. 2a).

SSEPs were obtained from the upper extremities using median nerve stimulation at a rate of $5.1 \mathrm{~Hz}$, with a stimulus duration of $0.2 \mathrm{~ms}$. There was a lack of response of SSEPs in the left upper extremity, including Erb's point potential.

\section{EMG/NCV of the Left Hand}

The muscles tested by needle EMG included the APB, FPL, FDP, and pronator teres. Only the FPL and FDP showed a decrease in motor unit recruitment. No fibrillations or positive 


\section{Case Reports in Neurology}
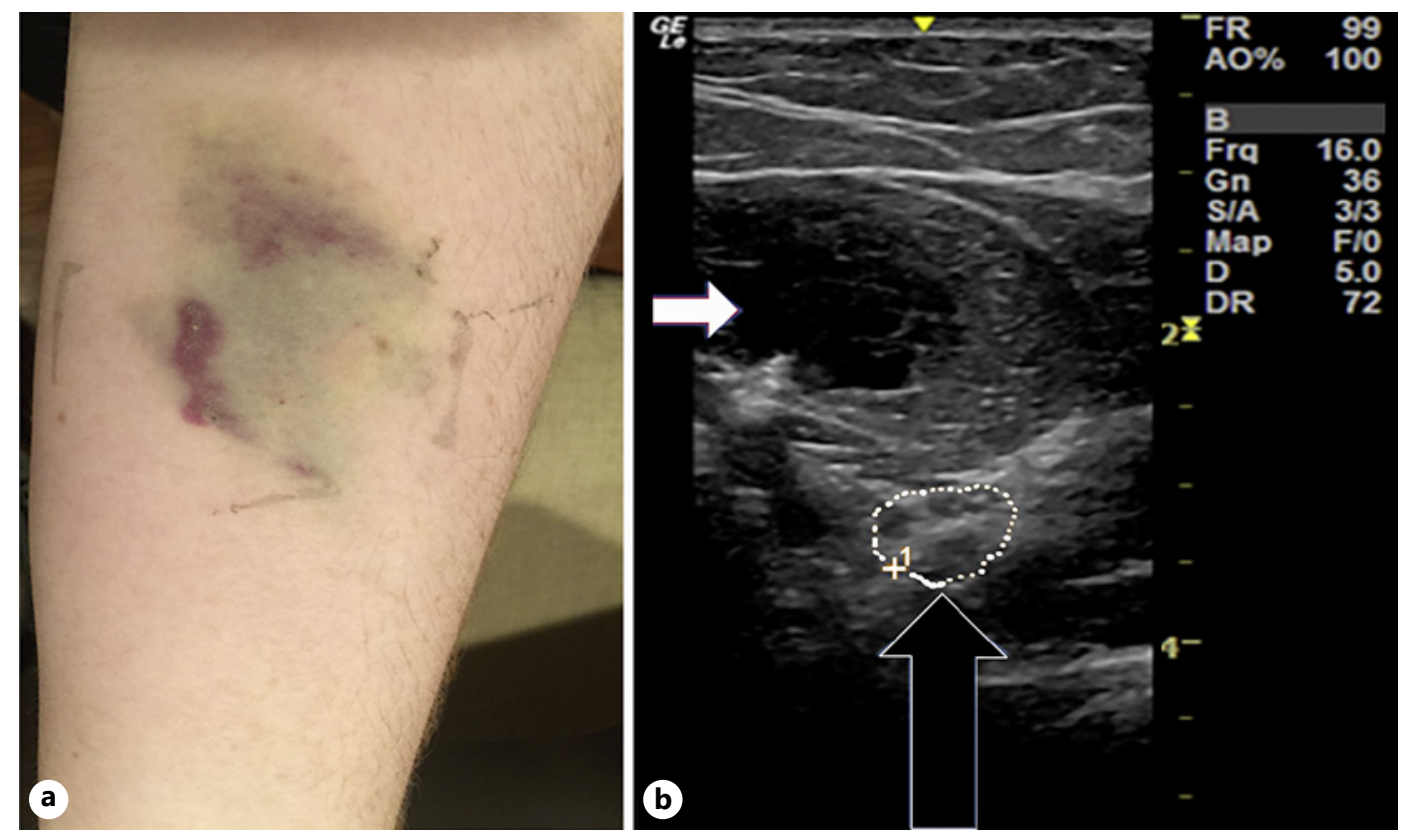

Fig. 2. a Ecchymosis on the volar aspect of the proximal left forearm 1 week postoperatively. $\mathbf{b}$ US at antecubital fossa (short axis view) demonstrating an increase in the CSA of the left median nerve (CSA of $37 \mathrm{~mm}^{2}$ compared to normal of $<10 \mathrm{~mm}^{2}$ ) 2 weeks postoperatively (black arrow). White arrow indicates the hematoma. CSA, cross-sectional area; US, ultrasound.

sharp waves were seen. The study was performed 10 days after the onset of symptoms; hence, it may have been too early to see fibrillations. Nevertheless, the patient was able to volitionally recruit several motor units in both FPL and FDP, which suggests partial conduction block or a mild axonal injury. Repeating the study 3-4 weeks later may have demonstrated fibrillation potentials. The motor NCV was performed with the recording electrode over the APB which did not show evidence for conduction block. Documentation of conduction block selectively in AIN fascicles is difficult/unreliable and, therefore, was not attempted. There was evidence for some sensory axonal involvement based on the decreased amplitude of digital sensory nerve action potentials on stimulation at the wrist (distal to the injury). It is most likely that the injury was partial and resulted in a combination of conduction block in a few motor fascicles (supplying the AIN) and axonal injury in a few sensory fascicles (input from digits 3 and 4).

US showed an increased cross-sectional area ( $37 \mathrm{~mm}^{2}$ compared to normal of $<10 \mathrm{~mm}^{2}$ ) of the left median nerve at the proximal forearm (Fig. 2b). EMG/NCS studies confirmed a left median nerve neuropathy at the level of the proximal forearm at the origin of the AIN. SSEPs of the left upper extremity were normal 3 weeks later. The intraoperative SSEP changes may have been positional, but her symptoms most likely resulted from median nerve compression by direct injury to the median nerve from phlebotomy which also caused the loss of SSEP waveforms.

\section{Follow-Up}

The patient was prescribed gabapentin $300 \mathrm{mg}$ twice daily which was increased to 600 $\mathrm{mg}$ twice daily without any benefit. She was also continued on topiramate $150 \mathrm{mg}$ two daily, oxcarbazepine $600 \mathrm{mg} 3$ times daily, and memantine $10 \mathrm{mg}$ twice daily as part of her treatment regimen for her chronic facial pain. The patient was referred to physical therapy but was

\section{Karger'}


unable to continue therapy due to financial constraints. The patient was prescribed a TENS unit that was applied to the palmar and dorsal aspects of the left hand to decrease her finger symptoms and to the wrist to reduce her thumb and thenar eminence symptoms. The patient used the TENS unit continuously for 2 months with a significant benefit.

At 5 weeks postoperatively, she was referred to an interventional pain management specialist for further treatment. The patient was started on nortriptyline $25 \mathrm{mg}$ daily and scheduled for a left stellate ganglion block under US and fluoroscopic guidance. Following the injection, the patient received $45-50 \%$ sustained relief of her painful symptoms. Of note, she continued to complain of numbness and slight motor weakness. A second left stellate ganglion block was performed 2 weeks after the initial procedure. The patient attained near-complete resolution of pain 2 weeks after the second block. She was able to discontinue nortriptyline and gabapentin. Some numbness continued to persist in the median nerve distribution.

Within 5 months of symptom onset, she experienced complete resolution of her left hand weakness, with continued numbness to temperature and texture of the radial left 3 fingers and radial half of the ring finger. This numbness persisted 10 months later.

Seven weeks following her second craniectomy, the patient underwent a left percutaneous trigeminal balloon compression gangliolysis. This led to approximately 6 months of satisfactory pain relief, and at the time of writing, the patient is considering repeat open exploration.

\section{Discussion}

Generally, it is thought that perioperative peripheral injury is more likely due to traction palsy, rather than venipuncture-related injury. Moore and colleagues [4] reviewed 313 iatrogenic nerve injuries from operative and nonoperative claims accepted by a no-fault compensation scheme in New Zealand. The most common causes of iatrogenic nerve injury were malpositioning (40 cases [12.8\%]), followed by venipuncture (26 cases [8.3\%]). Of the 40 cases of positional neuropraxia, 36 occurred in the operating room (35 under general anesthesia), 3 in the intensive care unit under sedation and/or paralysis, and 1 in an acute care ward. The median nerve was damaged in 18 (69.2\%) cases. Of these 26 were venipuncturerelated. Iatrogenic nerve injuries associated with venipuncture occurred in laboratories $(n=$ $18)$, general practice $(n=5)$, emergency rooms $(n=2)$, and the ward $(n=1)$. An iatrogenic brachial plexus injury was noted in $26(8.3 \%)$ of the 313 cases.

In their study of 587,551 venipuncture occurrences in the upper extremities, Kato and colleagues [5] reported that 19 patients experienced neuropathic pain; all of them experienced complete recovery within 6 months. The median nerve was affected in 2 patients in their study; both of them experienced pain in the cubital area with hypesthesia in the thumb, index, and middle fingers. The median antebrachial vein was punctured in both cases. Kochanowicz and colleagues [6] described 2 cases of median nerve damage caused by a routine intravenous infusion. One patient underwent infusion of $1 \%$ lidocaine, while the other had aminophylline infusion. Both experienced severe pain in the antecubital fossa radiating to the shoulder and forearm immediately after infusion, with clinical signs of sensorimotor fiber damage in the median nerve distribution. Proximal median nerve damage at the level of the antecubital fossa was confirmed electrophysiologically. The authors hypothesize that the mechanism was pressure-related compartment syndrome due to infusion of drugs into the musculofascial compartment of the median nerve. However, they acknowledge that drugrelated toxicity could not be excluded.

US can be a useful adjunct for the venipuncture procedure. In Ohnishi and colleagues' [10] study of US use to determine the location of the median nerve in relation to major blood vessels in the antecubital fossa, the authors describe that the median distance between the 
Fig. 3. Diagram depicting placement of an additional recording electrode over the proximal upper arm during intraoperative SSEP monitoring. SSEPs, somatosensory evoked potentials.

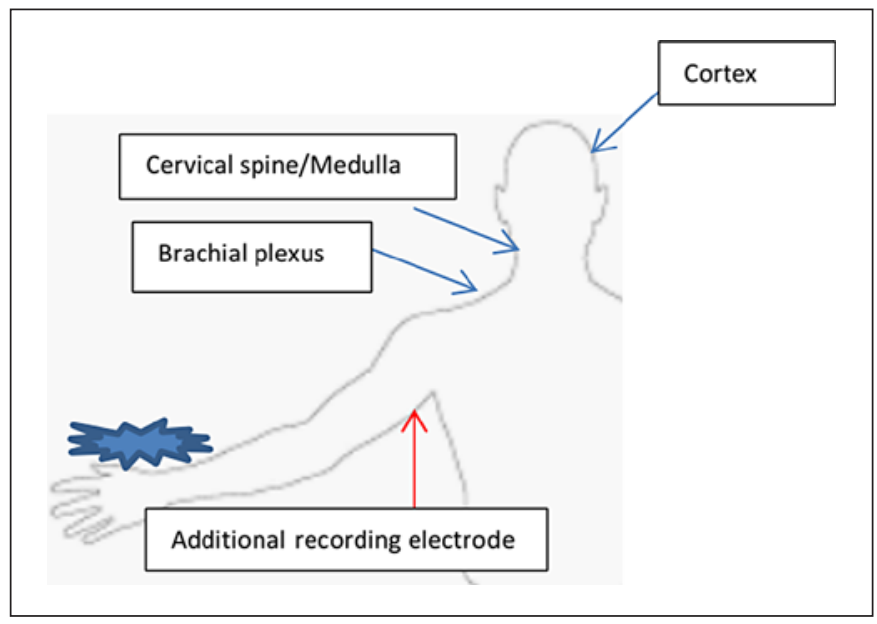

median nerve and the brachial artery was $1.4 \mathrm{~mm}$ at the elbow crease. They recommended that phlebotomists palpate the brachial artery prior to venipuncture and avoid puncture within 1 finger-width ulnar to the artery in the antecubital fossa.

Thorough history and neurological examinations are warranted in patients who complain of burning, paresthesia, and/or weakness following venipuncture, especially if there is evidence of ecchymosis in the antecubital fossa. Electrodiagnostic studies and US are valuable tests to elucidate the location of nerve injury. In neuropraxia, EMG may be normal or show decreased recruitment. In injuries resulting in axonal loss, EMG may be normal for the first 10-14 days after injury. EMG will then start to demonstrate abnormalities such as positive sharp waves and fibrillations [11]. In cases of isolated neuropraxia, NCS will show absent motor responses immediately. In axonal injury (axonotmesis), motor conduction studies will demonstrate the same pattern as neuropraxia until Wallerian degeneration has occurred. This process typically happens approximately 9 days following injury. Neuropraxia and axonotmesis become distinguishable when the motor response falls both proximal and distal to the lesion [11]. EMG/NCS studies should be used concomitantly to more fully define the characteristics of the injury including localization, age of injury, severity of injury, prognosis, and recovery [12]. Whereas radiographic studies may have a limited role in diagnosing peripheral nerve injuries, they can be useful when correlated with EMG/NCS. High-resolution MRI and US can be utilized in peripheral nerve imaging and may be helpful in isolating the exact site of a lesion [12]. Particular attention should focus on iatrogenic causes of neuropraxia, such as brachial plexus and peripheral nerve injuries, that may develop during surgical intervention.

Careful consideration should be made for patients undergoing venipuncture, especially in an anesthetized patient. In instances where cannulation cannot be performed with patient feedback, the use of US guidance should be considered. There are emerging data and recommendations on the benefits of US utilization in the peripheral venipuncture, especially in difficult and high-risk patients [13-15].

Routine SSEP monitoring stimulates the median nerve at the wrist and uses recording electrodes over the brachial plexus, upper cervical spine, and scalp. If the evoked potentials are lost over all of these sites, the pathology may be anywhere at or distal to the brachial plexus.

We recommend placing an additional recording electrode over the proximal upper arm during intraoperative SSEP monitoring to distinguish between a peripheral lesion (such as in the antecubital fossa) and a brachial plexus lesion (Fig. 3). If the lesion is located in the brachial plexus, the potential over the upper arm electrode will be intact, whereas all potentials will be lost if the lesion is at the antecubital fossa. This will expedite diagnosis of the specific iatrogenic 
nerve injury. We also suggest that arterial/venous access should be performed under US guidance when a patient is anesthetized and unable to give sensory feedback from a venipuncture needle contacting a peripheral nerve.

\section{Conclusion}

All surgeons, neurologists, and anesthesiologists should be aware that a median nerve injury may develop following venipuncture to the antebrachial fossa. SSEPs, EMG/NCS, and US can aid in performing the diagnosis. Treatment with neuropathic pain medications, TENS, and interventional pain management techniques may be helpful, and most cases resolve over the following months. Prompt recognition and treatment of an iatrogenic median neuropathy offer an excellent prognosis for symptomatic improvement.

\section{Acknowledgement}

We acknowledge Norton Healthcare for their continued support.

\section{Statement of Ethics}

Written informed consent was obtained from the patient for publication of this case report and any accompanying images. The University of Louisville Institutional Review Board has determined that our project does not meet the "Common Rule" definition of human subjects' research and does not require IRB review. The Institutional Review Board number is 19.1254 .

\section{Conflict of Interest Statement}

The authors have no conflicts of interest to declare.

\section{Funding Sources}

The authors did not receive any funding.

\section{Author Contributions}

Lisa B.E. Shields: data conception, design, acquisition, analysis, and interpretation; drafted the manuscript; critically revised the manuscript; and gave final approval. Brandon Sutton: data conception, design, acquisition, analysis, and interpretation; critically revised the manuscript; and gave final approval. Vasudeva G. Iyer: data conception, design, acquisition, analysis, and interpretation; critically revised the manuscript; and gave final approval. Christopher B. Shields: data conception, design, acquisition, analysis, and interpretation; critically revised the manuscript; and gave final approval. Abigail J. Rao: data conception, design, acquisition, analysis, and interpretation; critically revised the manuscript; and gave final approval.

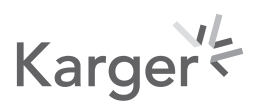




\section{References}

1 Antoniadis G, Kretschmer T, Pedro MT, Konig RW, Heinen CP, Richter HP. Iatrogenic nerve injuries: prevalence, diagnosis and treatment. Dtsch Arztebl Int. 2014;111:273-9.

2 Kretschmer T, Antoniadis G, Braun V, Rath SA, Richter HP. Evaluation of iatrogenic lesions in 722 surgically treated cases of peripheral nerve trauma. J Neurosurg. 2001;94:905-12.

3 Welch MB, Brummett CM, Welch TD, Tremper KK, Shanks AM, Guglani P, et al. Perioperative peripheral nerve injuries: a retrospective study of 380,680 cases during a 10-year period at a single institution. Anesthesiology. 2009;111:490-7.

4 Moore AE, Zhang J, Stringer MD. Iatrogenic nerve injury in a national no-fault compensation scheme: an observational cohort study. Int J Clin Pract. 2012;66:409-16.

5 Kato J, Araki H, Kimura M, Takahashi K, Ueda K, Iida R, et al. Incidence and prognosis of persistent pain induced by venipuncture for blood sampling: an observational study over a 5-year period. Pain Med. 2012;13:1627-30.

6 Kochanowicz J, Borkowski J, Borowik H. Venopuncture related median nerve damage. Case Rep Clin Pract Rev. 2005;6:41-3.

7 Newman BH, Waxman DA. Blood donation-related neurologic needle injury: evaluation of 2 years' worth of data from a large blood center. Transfusion. 1996;36:213-5.

8 Oven SD, Johnson JD. Radial nerve injury after venipuncture. J Hand Microsurg. 2014;9:43-4.

9 Elahi F, Reddy CG. Venipuncture-induced complex regional pain syndrome: a case report and review of the literature. Case Rep Med. 2014;2014:613921.

10 Ohnishi H, Urata T, Kishino T, Takano M, Watanabe T. A novel maneuver to prevent median nerve injury in phlebotomy. Ann Intern Med. 2009;151:290-1.

11 Winn HR. Youmans neurological surgery. 6th ed. Atlanta: Elsevier Sounders; 2011.

12 Lalkhen AG, Bhatia K. Perioperative peripheral nerve injuries. Contin Educ Anaesth Crit Care Pain. 2012; 12(1):38-42.

13 American Institute of Ultrasound in Medicine. AIUM practice guideline for the use of ultrasound to guide vascular access procedures. J Ultrasound Med. 2013;32:191-215.

14 Troianos CA, Hartman GS, Glas KE, Skubas NJ, Eberhardt RT, Walker JD, et al. Special articles: guidelines for performing ultrasound guided vascular cannulation: recommendations of the American Society of Echocardiography and the Society Of Cardiovascular Anesthesiologists. Anesth Analg. 2012;114:46-72.

15 Weiner MM, Geldard P, Mittnacht AJ. Ultrasound-guided vascular access: a comprehensive review. J Cardiothorac Vasc Anesth. 2013;27:345-60. 